\title{
The Existence of the Thermodynamic Limit in Coulomb-like Systems
}

\author{
Joseph Neely Gregg, Jr.
}

Department of Mathematics, Columbia University, New York, New York 10027, USA

\begin{abstract}
A Coulomb-like system is a system in which the usual $1 / r$ Coulomb potential has been replaced by a potential that goes as $1 / r^{\alpha}$ for $\alpha$ near one. Such potentials do not have the mean value property, which forms the basis of the Lebowitz-Lieb argument to control the long range Coulomb interaction [5], so whether or not such systems actually exhibit thermodynamic behavior is an interesting question. In this paper I generalize the proof of the limit for a crystal with Coulomb potential given by Charles Fefferman [1] to cover these Coulomb-like potentials.
\end{abstract}

\section{Introduction}

The statistical mechanics of systems of electrons and protons interacting via the Coulomb interaction has long been a subject of interest. One would like to know a little more about systems with a pair interaction that goes as some power $\alpha \neq 1$ of the inverse distance simply to answer the question "In what sense is the Coulomb potential special?" In this paper I hope to demonstrate that the Coulomb potential $(\alpha=1)$ is not special, at least in the sense that as one moves away from $\alpha=1$ nothing catastrophic happens and the resulting systems still have a thermodynamic limit.

First, the basic set-up. The states of our system of $N_{1}$ electrons and $N_{2}$ protons are taken to be the eigenfunctions of the quantum mechanical Hamiltonian

$$
H_{N, B_{R}}=\sum_{j=1}^{N_{1}}-\kappa_{1} \Delta_{x_{j}}+\sum_{k=1}^{N_{2}}-\kappa_{2} \Delta_{y_{k}}+\frac{1}{2} \sum_{j \neq 1} \frac{1}{\left|x_{j}-x_{l}\right|^{\alpha}}+\frac{1}{2} \sum_{k \neq m} \frac{1}{\left|y_{k}-y_{m}\right|^{\alpha}}-\sum_{j, k} \frac{1}{\left|x_{j}-y_{k}\right|^{\alpha}}
$$

with Dirichlet boundary conditions on some large ball $B_{R}$. The eigenfunctions $\psi\left(x_{1}, \ldots, x_{N 1}, y_{1}, \ldots, y_{N 2}\right)$ are assumed to all be $L^{2}$ functions that are separately antisymmetric in the $x$ and $y$ variables. In the following the appropriate $L^{2}$ space will be denote $L^{2}\left(\left(B_{R}\right)^{N 1+N 2}\right)$ or $L_{N}^{2}\left(B_{R}\right)$. The operator we have written down is self-adjoint for $0<\alpha<2$ (see, e.g. Reed \& Simon [6]), so we will consider that range of $\alpha$ in the following.

For convenience we will consider only the grand canonical ensemble: a 
given eigenstate of $N_{1}$ electrons and $N_{2}$ protons appears with probability $\exp (\mu \cdot N-\beta E(\psi)) / Z\left(\mu, \beta, B_{R}\right)$, where $E(\psi)=\left\langle H_{N, B_{R}} \psi \mid \psi\right\rangle$ and $Z\left(\mu, \beta, B_{R}\right)=$ $\sum_{N_{1}, N_{2} \geqq 0} \operatorname{Tr}\left[\exp \left(\mu \cdot N-\beta H_{N, B_{R}}\right)\right]$.

The subject of this paper is a proof of

The Existence of the Thermodynamic Limit for Coulomb-like Systems. The quantity

$$
\Pi_{R}(\beta, \mu)=\frac{1}{\left|B_{R}\right|} \ln \left\{\sum_{N} e^{\mu \cdot N} \operatorname{Tr}\left[\exp \left(-\beta H_{N, B_{R}}\right)\right]\right\}
$$

tends to a finite limit as $R \rightarrow \infty$.

The limit is a pointwise limit, but since the functions $\Pi_{R}$ are convex and can easily be shown to be bounded the limit is actually uniform on compact sets.

The common method in most such proofs of the existence of the infinite volume pressure limit is to compare the original large system with a system composed of a number of sub-components and demonstrate that the strength of the interactions between the components is relatively small compared to the strength of the interactions among the particles in a given component [7]. The original proof for Coulomb systems, due to Lebowitz and Lieb, made clever use of the mean value property of the Coulomb potential; namely, any neutral, spherically symmetric distribution of charge does not induce a potential at a distance. The idea of the Lebowitz-Lieb proof was to cover a large region very efficiently with a large number of disjoint balls of various sizes and create a system of neutral collections of particles lying in the balls. They showed that it is possible to approximate the actual $Z$ very closely with the $Z$ that comes from the system of balls and thus demonstrate the existence of the thermodynamic limit [5]. This clever use of the mean value property allows one to overcome the problems associated with the long range character of the Coulomb potential.

More recently, Charles Fefferman has given an alternative proof of the existence of the limit for Coulomb systems [1]. Fefferman looks at a system of nuclei arranged in a lattice, such as one would find in a crystal. This arrangement is not rotationally invariant, so the original method of Lebowitz and Lieb fails. The method used is to compare the original system with a "phony" system of non-interacting subcomponents. One makes this comparison by creating a set of injection operators that map a simple Hamiltonian defined on the phony system to an operator $H_{N, B_{R}}^{\#}$ on the original Hilbert space and showing that the differences between $H_{N, B_{R}}^{\#}$ and $H_{N, B_{R}}$ are small.

The proof in the present case is based on Fefferman's proof for crystals. Although one can follow the same arguments fairly closely on the whole, it is necessary to make a number of modifications of the original proof. The most important modification that one needs occurs in the places where Fefferman makes use of the mean value property. The most important instance of this modification comes when one tries to get some control on the effects of the potential at short range: one can replace Fefferman's original arguments in that case with a proof based on some ideas developed by Fefferman and Llave in their study of the relativistic stability of matter problem [3]. Other modifications are necessary for technical reasons based on the fact that the exponent in the potential is not one. Two such 
modifications are the introducion of an average over the sizes of the balls found in the decomposition of the large system and a slightly modified proof of the main lemma below.

\section{The Main Lemma}

As stated above, the idea of the proof of the thermodynamic limit is to compare the actual Hamiltonian with a modified Hamiltonian that comes from an "exploded" system. The most difficult portion of the analysis is the comparison of the actual potential energy with the "phony" potential energy that appears in the modified Hamiltonian. Our resource for dealing with this problem is the main lemma proved in this section. This lemma is of some interest in its own right as a method for comparing Hamiltonians with similar potential energies:

Main Lemma. Given an even function $k(x)$ satisfying

$$
\left|\partial_{x}^{\beta} k(x)\right| \leqq C(\alpha, \beta)|x|^{-\alpha-\beta} \quad \text { for } \quad|\beta| \leqq 3 \text { and all } x,
$$

the potential that $k(x)$ gives rise to,

$$
V[k(x)]=\frac{1}{2} \sum_{i \neq j} e_{i} e_{j} k\left(x_{i}-x_{j}\right)
$$

satisfies

$$
V[k(x)] \leqq C\left(H_{N, B_{R}}+C\left(N_{1}+N_{2}\right)\right)
$$

in the sense of operators on $L_{N}^{2}\left(B_{R}\right)$.

Proof. First of all, (1) says that $k(x)$ is like $|x|^{-\alpha}$ in a certain special sense. One useful way to express this "likeness" is via the Fourier transform. The Fourier transform of $|x|^{-\alpha}$ looks like (Const) $|\xi|^{\alpha-3}$. The Fourier transform of $k$ is similar in that it satisfies $|\hat{k}(\xi)| \leqq C|\xi|^{\alpha-3}$. To see this, write $k(x)=k_{1}(x)+k_{2}(x)$ with both $k_{1}$ and $k_{2}$ even functions satisfying (1). Suppose further that we fix some $\xi^{\prime}$ and require that $k_{1}(x)$ be supported in $|x| \leqq 2\left|\xi^{\prime}\right|^{-1}$ and $k_{2}(x)$ be supported in $|x| \geqq\left|\xi^{\prime}\right|^{-1}$.

First note that

$$
\left\|k_{1}\right\|_{L^{1}} \leqq C(\alpha) \int_{|x|<2\left|\xi^{\prime}\right|^{-1}}|x|^{-\alpha} d^{3} x=C(\alpha) 4 \pi \int_{0}^{2\left|\xi^{\prime}\right|^{-1}} r^{2-\alpha} d r=\frac{C(\alpha) 4 \pi}{3-\alpha}\left(2\left|\xi^{\prime}\right|^{-1}\right)^{3-\alpha}
$$

This implies that the Fourier transform satisfies $\left|\hat{k}_{1}(\xi)\right| \leqq C^{\prime}(\alpha)\left|\xi^{\prime}\right|^{\alpha-3}$ for our one special fixed value of $\xi$.

The second function, $k_{2}(x)$, is supported away from the origin, so the same argument will not work. Instead, we use the derivatives of $k_{2}(x)$ to control its Fourier transform. We claim

$$
\int\left|\Delta k_{2}(x)-\Delta k_{2}(x-y)\right| d^{2} x<C(\alpha)\left|\xi^{\prime}\right|^{\alpha-1}
$$

for $|y|<c_{1}\left|\xi^{\prime}\right|^{-1}, c_{1} \ll 1$. Taylor expanding $\Delta k_{2}(x-y)$ to first order in $y$ gives

$$
\Delta k_{2}(x-y)=\Delta k_{2}(x)+\sum_{|\beta|=1} \frac{\partial_{x}^{\beta} \Delta k_{2}(x-Y)}{\beta !} y^{\beta}
$$


for some $Y$ in $|y|<c_{1}\left|\xi^{\prime}\right|^{-1}$. Equation (1) together with the fact that $k_{2}(x)$ has support in $|x| \geqq\left|\xi^{\prime}\right|^{-1}$ give $\left|\partial_{x}^{\beta} \Delta k_{2}(x-Y)\right| \leqq\left.\left. C|| x\left|-c_{1}\right| \xi^{\prime}\right|^{-1}\right|^{-3-\alpha}$ for $|\beta|=1$ so

$$
\begin{aligned}
\int\left|\Delta k_{2}(x)-\Delta k_{2}(x-y)\right| d^{3} x & \leqq\left.\left. C \int_{|x|>\left|\xi^{\prime}\right|-1}|| x\left|-c_{1}\right| \xi^{\prime}\right|^{-1}\right|^{-3-\alpha}\left|\xi^{\prime}\right|^{-1} d^{3} x \\
& \leqq C \int_{|x|>\left(1-c_{1}\right)\left|\xi^{\prime}\right|-1}|x|^{-3-\alpha}\left|\xi^{\prime}\right|^{-1} d^{3} x \\
& =C\left|\xi^{\prime}\right|^{-1} \int_{r=\left.\left(1-c_{1}\right)\left|\xi^{\prime}\right|\right|^{-1}} r^{-1-\alpha} d r=C(\alpha)\left|\xi^{\prime}\right|^{-1+\alpha} .
\end{aligned}
$$

Comparing the Fourier transform of $\Delta k_{2}(x)-\Delta k_{2}(x-y)$ with the left-hand side of (2) and noting that in particular one is free to set $\xi=\xi^{\prime}$ gives

$$
\left.\left.\left|\left[1-e^{i v \cdot \xi^{\prime}}\right]\right| \xi^{\prime}\right|^{2} k_{2}\left(\xi^{\prime}\right)|\leqq C(\alpha)| \xi^{\prime}\right|^{\alpha-1} .
$$

Setting $y=\left(c_{1} / 2\right) \xi^{\prime} \cdot\left|\xi^{\prime}\right|^{-2}$ in (3) gives the inequality $\left|\hat{k}_{2}\left(\xi^{\prime}\right)\right| \leqq C^{\prime}(\alpha)\left|\xi^{\prime}\right|^{\alpha-3}$.

Putting the inequalities for the transforms of $k_{1}(x)$ and $k_{2}(x)$ together yields the inequality: $\left|\hat{k}\left(\xi^{\prime}\right)\right| \leqq\left|\hat{k}_{1}\left(\xi^{\prime}\right)\right|+\left|\hat{k}_{2}\left(\xi^{\prime}\right)\right| \leqq C\left|\xi^{\prime}\right|^{\alpha-3}$. Finally, note that the constants we have been writing down have not depended on our choice of $\xi^{\prime}$ : they depend at most on the constants in (1), $\alpha$, and on the geometry of the situation. That means that the desired inequality holds independent of our choice of $\xi^{\prime}$.

Now consider a new potential $k^{\#}(x)=|x|^{-\alpha}-c k(x)$, where $c \ll 1$. The constant $c$ will be chosen small enough to guarantee that $k^{\#}(x)$ has positive Fourier transform. We will compare $V\left[k^{\#}(x)\right]$ with a continuous version obtained by picking a function $\phi(x) \in C_{0}^{\infty}(B(0,1 / 3))$ such that $\int \phi(x) d^{3} x=1$ and $\int x^{\gamma} \phi(x) d^{3} x=0$ for $0<|\gamma|<10$, setting $\phi_{j}(x)=\left[\delta\left(z_{j}\right)\right]^{-3} \phi\left(x / \delta\left(z_{j}\right)\right)$, where the operator $\delta\left(z_{j}\right)$ is the distance from the particle at $z_{j}$ to its nearest neighbor, and writing $\rho(x)=\sum_{j} e_{j} \phi_{j}\left(x-z_{j}\right)$. One then constructs the smoothed out potential $k^{\sharp}\{\rho\}=\frac{1}{2} \int k^{\#}(x-y) \rho(x) \rho(y) d^{3} x d^{3} y$.

Because $k^{\#}$ has positive Fourier transform the smoothed out potential operator is a positive operator. Hence if we can control the difference between $V\left[k^{\sharp}\right]$ and $k^{\#}\{\rho(x)\}$ we can control $V\left[k^{\#}\right]$.

The difference can be written as a sum over pairs of particles of terms

$$
\left|k^{\#}\left(z_{j}-z_{k}\right)-k^{\#} * \phi_{j} * \phi_{k}\left(z_{j}-z_{k}\right)\right|
$$

plus a sum of self-energy terms with size bounded by $C \sum_{j}\left[\delta\left(z_{j}\right)\right]^{-\alpha}$.

Taylor expanding $k^{\#}(x)$ to order three in (4) and using the moment properties of the $\phi_{j}$ to eliminate the lower order terms gives an upper bound of

$$
\frac{C\left(\delta\left(z_{j}\right)+\delta\left(z_{k}\right)\right)^{3}}{\left|z_{j}-z_{k}\right|^{3+\alpha}}
$$

for the difference. We can control (5) in turn by introducing the functions

$$
\begin{aligned}
& F(x)=\sum_{j}\left[\delta\left(z_{j}\right)\right]^{-3} \chi\left(\left|x-z_{j}\right|<\frac{\delta\left(z_{j}\right)}{3}\right), \\
& G(x)=\sum_{j}\left[\delta\left(z_{j}\right)\right]^{-\alpha} \chi\left(\left|x-z_{j}\right|<\frac{\delta\left(z_{j}\right)}{3}\right) .
\end{aligned}
$$


Now

$$
\begin{aligned}
\sum_{j, k} \frac{\left[\delta\left(z_{j}\right)\right]^{3}}{\left|z_{j}-z_{k}\right|^{3+\alpha}} & \leqq 2^{3+\alpha} \sum_{j, k} \frac{\left[\delta\left(z_{j}\right)\right]^{3}}{\left(\delta\left(z_{j}\right)+\left|z_{j}-z_{k}\right|\right)^{3+\alpha}} \\
& \leqq C \sum_{j} \int_{\left|x-z_{j}\right|<\delta\left(z_{j}\right) / 3} G(x)\left[\int_{\mathbb{R}^{3}} \frac{\left[\delta\left(z_{j}\right)\right]^{\alpha}}{\left(\delta\left(z_{j}\right)+|x-y|\right)^{3+\alpha}} F(y) d^{3} y\right] d^{3} x \\
& \leqq C \sum_{j} \int_{\left|x-z_{j}\right|<\delta\left(z_{j}\right) / 3} G(x) F^{*}(x) d^{3} x \\
& =C \int_{\mathbb{R}^{3}} G(x) F^{*}(x) d^{3} x,
\end{aligned}
$$

where $F^{*}(x)$ is the maximal function of $F$ (see Stein [8]). The Maximal Theorem allows us to bound the last integral from above by

$$
C\|G\| L^{(\alpha+3) / \alpha}\|F\| L^{(\alpha+3) / 3} \leqq C \sum_{j}\left[\delta\left(z_{j}\right)\right]^{-\alpha} .
$$

The same argument with $z_{k}$ in place of $z_{j}$ gives

$$
\sum_{j, k} \frac{\left[\delta\left(z_{k}\right)\right]^{3}}{\left|z_{j}-z_{k}\right|^{3+\alpha}} \leqq C \sum_{h}\left[\delta\left(z_{k}\right)\right]^{-\alpha} .
$$

Clearly $\sum_{j, k}\left|k^{\#}\left(z_{j}-z_{k}\right)-k^{\#} * \phi_{j} * \phi_{k}\left(z_{j}-z_{k}\right)\right| \leqq C \sum_{j}\left[\delta\left(z_{j}\right)\right]^{-\alpha}$. This equation combined with the fact that $k^{\#}\{\rho\} \geqq 0$ gives

$$
V\left[k^{\#}\right] \geqq-C \sum_{j}\left[\delta\left(z_{j}\right)\right]^{-\alpha} .
$$

The next section contains a proof of the inequality

$$
\sum_{j}\left[\delta\left(z_{j}\right)\right]^{-\alpha} \leqq\left(H_{N, B_{R}}+C(\alpha)\left(N_{1}+N_{2}\right)\right) .
$$

The inequalities (6) and (7) together with the definition of $k^{\#}(x)$ yield

$$
\begin{aligned}
V[k(x)] & \leqq C(\alpha)\left(H_{N, B_{R}}+C\left(N_{1}+N_{2}\right)\right)+C V\left[|x|^{-\alpha}\right] \\
& \leqq C^{\prime}(\alpha)\left(H_{N, B_{R}}+C\left(N_{1}+N_{2}\right)\right),
\end{aligned}
$$

and the main lemma is proved.

Note: From time to time in the following it will be necessary to apply the main lemma to - $K(x)$ instead of $K(x)$; in those cases the main lemma still applies and one gets inequalities like $V[-K(x)] \geqq-C\left(H_{N, B_{R}}+C\left(N_{1}+N_{2}\right)\right.$.

\section{Controlling the Potential at Short Range}

The proof of the main lemma reduces to showing the following operator inequality on $L_{N}^{2}\left(B_{R}\right)$ :

$$
\sum_{j}\left[\delta\left(z_{j}\right)\right]^{-\alpha} \leqq\left(H_{N, B_{R}}+C(\alpha)\left(N_{1}+N_{2}\right)\right)
$$

The proof of this inequality will make use of some ideas first seen in Feffermann and Llave [3]. For technical reasons it is actually more convenient to prove the 
inequality on $L_{N}^{2}\left(\mathbb{R}^{3}\right)$ and then view (1) as a special case of the more general inequality.

Note that the Hamiltonian contains kinetic energy terms for both protons and electrons: if we were to fix the protons at arbitrary locations $y_{1}, \ldots, y_{N 1}$ and prove

$$
\sum_{j}\left[\delta\left(z_{j}\right)\right]^{-\alpha} \leqq\left(\sum_{k}\left(-\kappa_{1} \Delta_{z_{k}}\right)+\frac{1}{2} \sum_{i \neq j} \frac{e_{i} e_{j}}{\left|z_{i}-z_{j}\right|^{\alpha}}+C(\alpha) N_{1}\right)
$$

with the kinetic energy of the $N_{1}$ electrons only, it is clear that (2) would imply the $L_{N}^{2}\left(\mathbb{R}^{3}\right)$ version of $(1)$.

The method of Fefferman and Llave works on operators that are homogeneous in dilations of the coordinate system; therefore, we will prove

$$
C(\alpha) \kappa(\alpha)\left(\sum_{k}\left(-\Delta_{x_{k}}\right)^{-\alpha / 2}\right)+\frac{1}{2} \sum_{i \neq j} \frac{e_{i} e_{j}}{\left|z_{i}-z_{j}\right|^{\alpha}} \geqq \frac{1}{2} \sum_{j}\left[\delta\left(z_{j}\right)\right]^{-\alpha}
$$

for $C(\alpha)$ some constant to be specified and $\kappa(\alpha)$ sufficiently large. The simple inequality (proved by the Fourier transform)

$$
\begin{aligned}
(-\Delta) \geqq & C(\alpha) \kappa(\alpha)(-\Delta)^{\alpha / 2}-\left[(2 \pi)^{\alpha} C(\alpha) \kappa(\alpha)\left(\frac{(2 \pi)^{\alpha} \alpha C(\alpha) \kappa(\alpha)}{8 \pi^{2}}\right)^{\alpha /(2-\alpha)}\right. \\
& \left.-4 \pi^{2}\left(\frac{(2 \pi)^{\alpha} \alpha C(\alpha) \kappa(\alpha)}{8 \pi^{2}}\right)^{2 /(2-\alpha)}\right]
\end{aligned}
$$

combined with (3) proves (2).

The method of Fefferman and Llave makes use of a continuous decomposition of space into balls $B(z, R)$ centered at $z \in \mathbb{R}^{3}$ with radii $R, 0<R<\infty$. The simple equalities

where

$$
\frac{1}{|x-y|^{\alpha}}=C_{1}(\alpha) \int_{R>0} \int_{z \in \mathbb{R}^{3}} \chi(x, y \in B(z, R)) \frac{d^{3} z d R}{R^{4+\alpha}},
$$

where

$$
C_{1}(\alpha)=\frac{\alpha(1+\alpha)(3+\alpha)}{2^{2+\alpha} \pi}
$$

and

$$
\left\langle(-\Delta)^{\alpha / 2} u \mid u\right\rangle=C_{2}(\alpha) \int_{\mathbb{R}^{3} \mathbb{R}^{3}} \frac{|u(x)-u(y)|^{2}}{|x-y|^{3+\alpha}} d^{3} x d^{3} y,
$$

$$
C_{2}(\alpha)=\frac{\alpha(1+\alpha) \Gamma(\alpha) \sin \left(\alpha \frac{\pi}{2}\right)}{4 \pi^{2}}
$$

will allow us to write the familiar kinetic and potential energy terms as integrals over these balls. Equation (5) is trivial to prove and (6) follows from the Fourier transform. Inserting (5) in (6) gives

$$
\begin{aligned}
\left\langle\sum_{k}\left(-\Delta_{x_{k}}\right)^{\alpha / 2} \psi \mid \psi\right\rangle= & C_{2}(\alpha) C_{1}(3+\alpha) \sum_{k} \int_{z \in \mathbb{R}^{3}} \int_{R>0 \mathbb{R}^{3 N_{1}-3}} \int_{B(z, R)}\left|\psi\left(\bar{x}, x^{\prime}\right)-\psi\left(\overline{\bar{x}}, x^{\prime}\right)\right|^{2} \\
& \cdot d^{3} \bar{x} d^{3} \overline{\bar{x}} d^{3 N_{1}-3} x^{\prime} \frac{d R d^{3} z}{R^{7+\alpha}} .
\end{aligned}
$$


Rather than use this complicated expression for the kinetic energy we will use the fact that on balls $B(z, R)$ containing more than one electron

$$
\int_{\mathbb{R}^{3 N_{1}-3}} \int_{B(z, R)}\left|\psi\left(\bar{x}, x^{\prime}\right)-\psi\left(\overline{\bar{x}}, x^{\prime}\right)\right|^{2} d^{3} \bar{x} d^{3} \overline{\bar{x}} d^{3 N_{1}-3} x^{\prime} \geqq \int_{\mathbb{R}^{3 N_{1}}}(N(z, R ; x)-1)_{+}|\psi(x)|^{2} d^{3 N_{1}} x,
$$

where $N(z, R ; x)$ is the number of electrons in $B(z, R)$ for a given value of $x_{1}, \ldots, x_{N_{1}}$ and the operation $(\cdot)_{+}$returns the argument for positive arguments and zero for negative arguments. The inequality follows from the antisymmetry of the wave function in the $x$ variables [3].

The potential energy can be written as a linear combination of terms like (5):

$$
\frac{1}{2} \sum_{i \neq j} \frac{e_{i} e_{j}}{\left|z_{i}-z_{j}\right|^{\alpha}}=\frac{C_{1}(\alpha)}{2} \int_{z \in \mathbb{R}^{3}} \int_{R>0}[M(M-1)+N(N-1)-2 M N] \frac{d R d^{3} z}{R^{4+\alpha}},
$$

where $M(z, R ; y)$ is the number of protons and $N(z, R ; x)$ the number of electrons in $B(z, R)$. The expression for the short range potential looks more complicated. For our purposes it will be sufficient to use the lower bound

$$
\begin{gathered}
-\frac{1}{2} \sum_{j}\left[\delta\left(z_{j}\right)\right]^{-\alpha} \geqq-\frac{C_{1}(\alpha)}{2} \int_{z \in \mathbb{R}^{3}} \int_{R>0}[M(\chi(N \geqq 1)+\chi(M \geqq 2)) \\
+N(\chi(N \geqq 2)+\chi(M \geqq 1))] \frac{d R d^{3} z}{R^{4+\alpha}} .
\end{gathered}
$$

What we will do now is to combine to short range potential with the ordinary potential to produce a Hamiltonian with an "augmented" potential,

$$
V_{\text {aug }}=V\left[|x|^{-\alpha}\right]-\frac{1}{2} \sum_{j}\left[\delta\left(z_{j}\right)\right]^{-\alpha} .
$$

$V_{\text {aug }}$ plus a kinetic energy $\kappa(\alpha) \cdot(-\Delta)^{\alpha / 2}$ will give us an "augmented" Hamiltonian to work with. The object then is to show that the new Hamiltonian is a positive operator. Inequality (7) and the two expressions for the potentials above can be combined to give

$$
\begin{aligned}
& \left\langle\left[C_{3}(\alpha) \kappa(\alpha)\left(\sum_{k}\left(-\Delta_{x_{k}}\right)^{\alpha / 2}\right)+\frac{1}{2} \sum_{i \neq j} \frac{e_{i} e_{j}}{\left|z_{i}-z_{j}\right|^{\alpha}}-\frac{1}{2} \sum_{j}\left[\delta\left(z_{j}\right)\right]^{-\alpha}\right] \psi \mid \psi\right\rangle \\
& \geqq \frac{C_{1}(\alpha)}{2}\left(\frac{3}{8 \pi} \kappa(\alpha) \sum_{k} \int_{z \in \mathbb{R}^{3}} \int_{R>0} \int_{x^{\prime} \in\left(\mathbb{R}^{3} \backslash B\right)^{N_{1}-1}} \int_{B(z, R)}\left|\psi\left(\bar{x}, x^{\prime}\right)-\psi\left(\overline{\bar{x}}, x^{\prime}\right)\right|^{2}\right. \\
& \left.\cdot d^{3} \bar{x} d^{3} \overline{\bar{x}} d^{3 N_{1}-3} x^{\prime} \frac{d R d^{3} z}{R^{7+\alpha}}+\int_{x \in \mathbb{R}^{3 N_{1}}} \int_{z \in \mathbb{R}^{3}} \int_{R>0} \Omega(z, R ; x)|\psi(x)|^{2} \frac{d R d^{3} z}{R^{4+\alpha}} d^{3 N_{1}} x\right)
\end{aligned}
$$

where

$$
C_{3}(\alpha)=\frac{6 \pi}{(4+\alpha)(6+\alpha) \Gamma(\alpha) \sin \left(\alpha \frac{\pi}{2}\right)}
$$

The operator $\Omega(z, R ; x)$ is simply the combination of appropriate things from 
each of the three expressions:

$$
\begin{aligned}
\Omega(z, R ; x)= & M(M-1)+N(N-1)-2 M N-N(\chi(M \geqq 1)+\chi(N \geqq 2)) \\
& -M(\chi(N \geqq 1)+\chi(M \geqq 2))+\kappa(\alpha)(N-1)_{+} .
\end{aligned}
$$

See Fefferman \& Llave [3] for the details of a proof of a similar estimate.

This rather complicated quadratic form must be radically simplified if we are to have any hope of understanding it. One simplification is to think of the entire structure as a sum of individual "atoms" based on the division of the form into parts, each of which is assigned in some clearly defined way to a given proton. Since the integrals above are integrals over balls $B(z, R)$ it is natural to have a scheme that distributes the contribution of an individual ball to one or more protons associated with the ball. Balls containing one proton will give their entire contribution to that proton. Balls containing more than one proton will divide their contributions equally among the protons in the ball. Finally, if a ball contains no protons we will simply throw that term away. This has the result of making the form less positive: for any $\kappa(\alpha)>0$ we have $\Omega(z, R ; x)=N(N-1)-N_{\chi}(N \geqq 2)+$ $\kappa(\alpha)(N-1)_{+} \geqq 0$ for balls having no protons.

Consider the set of all balls assigned to a given $y_{j}$. Those balls that have many electrons in them can be expected to have more energy than other balls; this is in fact the case. Those values of $z$ and $R$ for which $N(z, R) \geqq 2$ have

$$
\frac{\Omega(z, R ; x)}{M(z, R ; x)} \geqq e(\kappa)=2 \sqrt{\kappa-2}-7,
$$

while balls in which $N=0,1$ have $\Omega(z, R ; x) / M(z, R ; x) \geqq(M-1)-4 \chi(N>0)-$ $\chi(M \geqq 2)$.

Clearly the thing to do is to look at a given proton and treat all the terms with one or no electrons with care and replace all the terms with many electrons with the crude lower bound (8). The resulting quadratic form still contains an integral over all the other electron coordinates; in view of our crude estimate, we can without loss of generality fix the coordinates $x^{\prime} \in \mathbb{R}^{3 N_{1}-3}$ of the other electrons and only worry about a single electron. This has the virtue of replacing the complicated many electron problem with a somewhat more simple one electron problem. Since this discussion is centered on a given proton it is natural to introduce a change of variables from $(z, R)$ to $(w, R)$, where $y_{j}=z+R w$.

The problem now is to analyze a quadratic form which looks like a sum over nuclei of integrals over $w$ and $R$. For a given $y_{j}$ and $w$ this form is

$$
\begin{aligned}
Q(u)= & \frac{3 \kappa(\alpha)}{8 \pi} \int_{0}^{\bar{R}} \int_{x, y \in B(R)}|u(x)-u(y)|^{2} d^{3} x d^{3} y \frac{d R}{M(R) R^{4+\alpha}} \\
& +\int_{B(\bar{R})}|u(x)|^{2}\left\{\frac{e(\kappa)}{\alpha \bar{R}^{\alpha}}+\int_{0}^{\bar{R}}[(M(R)-1)-\chi(M \geqq 2)-4 \chi(x \in B(R))] \frac{d R}{R^{1+\alpha}}\right\} d^{3} x,
\end{aligned}
$$

where $\bar{R}$ is the smallest radius for which $N(w, R)>1$ and $M(R)=M(w, R)$ is a positive increasing integer-valued step function that counts the number of protons and thus depends on the positions of the other protons. Therefore, what we need to show is that for $\kappa(\alpha)$ sufficiently large $Q(u)$ is $\geqq 0$ for any choice of the function 
$M(R)$. This will finish the proof. (See Fefferman \& Llave [3] for the details of a similar reduction.)

The form $Q$ is homogeneous under dilations, so without loss of generality we can set $\bar{R}=1$. It is also enough to prove positivity only for the case $w=0$ (and hence $B(R)=\{x|| x \mid<R\}$ below) and for radial $u[3]$.

We are going to compare $Q$ with the following quadratic form:

$$
\Phi(u)=\frac{3 \alpha(3+\alpha)^{2}}{8 \pi(\alpha-3)^{2}}\left(\int_{0}^{\infty} \int_{|x|,|y|<R}|u(x)-u(y)|^{2} d^{3} x d^{3} y \frac{d R}{R^{4+\alpha}}-\int_{\mathbb{R}^{3}} \frac{|u(x)|^{2}}{|x|^{\alpha}} d^{3} x\right) .
$$

A Mellin transform argument shows that for $u$ radial, square integrable, and vanishing near 0 and $\infty$ one can make the substitution $u(x)=\int_{-\infty}^{\infty}|x|^{-(3-\alpha) / 2+i \gamma}$ $[\tilde{u}(\gamma)] d \gamma$ and see that the form becomes $\Phi(\tilde{u})=\int_{-\infty}^{\infty} m(\gamma)|\tilde{u}(\gamma)|^{2} d \gamma$ with $m(\gamma) \geqq 0$ for all $\gamma \cdot\left(\right.$ Explicitly, $m(\gamma)=\frac{3 \alpha(3+\alpha)^{2}}{8 \pi(3-\alpha)^{2}}(4 \pi)^{2}(2 \pi) \frac{2}{3+\alpha}\left(\frac{1}{3}-\frac{3+\alpha}{\left((3+\alpha)^{2} / 4\right)+\gamma^{2}}+\frac{1}{\alpha}\right)-$ $(4 \pi)(2 \pi)$.)

A little bit of manipulation will make (9) and (10) look more alike:

$$
\begin{aligned}
& \int_{B(1)}|u(x)|^{2} \int_{0}^{1}(M-1-\chi(M \geqq 2)) \frac{d R}{R^{1+\alpha}} d^{3} x \\
& \quad \geqq \int_{0}^{1}(M-1-\chi(M \geqq 2)) \int_{|x|<R}|u(x)|^{2} d^{3} x \frac{d R}{R^{1+\alpha}} \\
& \quad \geqq \int_{0}^{1}(M-1-\chi(M \geqq 2)) \frac{3}{8 \pi R^{3}} \int_{|x|,|y|<R}|u(x)-u(y)|^{2} d^{3} x d^{3} y \frac{d R}{R^{1+\alpha}} .
\end{aligned}
$$

Also, $\int_{0}^{1}-4 \chi(x \in B(R)) \frac{d R}{R^{1+\alpha}}=\frac{4}{\alpha}-\frac{4}{\alpha|x|^{\alpha}}$. Hence

$$
\begin{aligned}
Q(u) \geqq & \frac{3}{8 \pi} \int_{0 x, y \in B(R)}^{1}|u(x)-u(y)|^{2}\left(\frac{\kappa(\alpha)}{M(R)}+M-1-\chi(M \geqq 2)\right) d^{3} x d^{3} y \frac{d R}{R^{4+\alpha}} \\
& +\frac{1}{\alpha} \int_{B(1)}(e(\kappa)+4)|u(x)|^{2} d^{3} x-\frac{4}{\alpha} \int_{B(1)} \frac{|u(x)|^{2}}{|x|^{\alpha}} d^{3} x .
\end{aligned}
$$

Applying $\Phi(u) \geqq 0$ to functions $u$ supported in the unit ball gives

$$
\begin{aligned}
& 0 \leqq \frac{3 \alpha(3+\alpha)^{2}}{8 \pi(3-\alpha)^{2}} \int_{0|x|,|y|<R}^{1}|u(x)-u(y)|^{2} d^{3} x d^{3} y \frac{d R}{R^{4+\alpha}}-\int_{|x|<1} \frac{|u(x)|^{2}}{|x|^{\alpha}} d^{3} x \\
& +\frac{\alpha(3+\alpha)^{2}}{(3-\alpha)^{2}} \int_{1}^{\infty} \int_{|x|<R}|u(x)-\underset{x \in B(R)}{\operatorname{avg}} u(x)|^{2} d^{3} x \frac{d R}{R^{1+\alpha}} \\
& \leqq \frac{3 \alpha(3+\alpha)^{2}}{8 \pi(3-\alpha)^{2}} \int_{0}^{1} \int_{|x|,|y|<R}|u(x)-u(y)|^{2} d^{3} x d^{3} y \frac{d R}{R^{4+\alpha}}-\int_{|x|<1} \frac{|u(x)|^{2}}{|x|^{\alpha}} d^{3} x \\
& +\frac{(3+\alpha)^{2}}{(3-\alpha)^{2}} \int_{|x|<1}|u(x)|^{2} d^{3} x \text {. }
\end{aligned}
$$


Comparing (11) and (12) we see that (11) will be true provided

$$
\frac{1}{4}\left(\frac{\kappa(\alpha)}{M(R)}+M(R)-1-\chi(M(R) \geqq 2)\right) \geqq \frac{(3+\alpha)^{2}}{(3-\alpha)^{2}}
$$

and

$$
\frac{e(\kappa)}{4}+1=\frac{\sqrt{\kappa-2}}{2}-\frac{3}{4} \geqq \frac{(3+\alpha)^{2}}{(3-\alpha)^{2}} .
$$

These are satisfied for

$$
\kappa>\left(2 \frac{(3+\alpha)^{2}}{(3-\alpha)^{2}}+\frac{3}{2}\right)^{2}+2
$$

\section{The Swiss Cheese Decomposition}

We now arrive at the description of the particular construction we will use to set up our proof of the existence of the thermodynamic limit. As stated earlier, the proof is based on a decomposition of some large region of space into smaller, simpler regions. When dealing with radial potentials it is natural to consider decompositions of space that preserve the spherical symmetry of the problem. The original idea of Lebowitz and Lieb was to decompose regions in space into sets of disjoint balls with geometrically increasing radii, a so-called swiss cheese decomposition of space. The specific swiss cheese used in the present proof is a version of the original Lebowitz and Lieb decomposition due to Hughes [4]:

Covering Lemma. Let a sequence of radii $1 \leqq R_{1}<\cdots<R_{M}, R_{i+1}>15 R_{i}$, be given. Let $Q$ be a large cube, $|Q|>(12(M+5))^{3}\left|B_{R M}\right|$. There exists a family of disjoint balls, all contained in $Q$, having radii $R_{1}, \ldots, R_{M}$ such that for all $i$

$$
\frac{1}{M+6}<\frac{\mid Q \cap\left(\text { Balls of radius } R_{i}\right) \mid}{|Q|}<\frac{1}{M+5} .
$$

Proof by Induction. Suppose $c_{i}$ balls of size $R_{i}$ have been packed into $Q$ for $i=j+1, \ldots, M$ where $c_{i} \in\left(|Q| /\left[(M+6)\left|B_{R i}\right|\right],|Q| /\left[(M+5)\left|B_{R i}\right|\right]\right)$. Let $\Omega^{j}$ be the complement of the set of balls that have already been placed in $Q$ for $i>j$. Since it is trivial to place $c_{M}$ disjoint balls of radius $R_{M}$ into a cube $Q$ with size $|Q|>2 M^{2}\left|B_{R M}\right|$, the lemma will be proved if we can show that it is possible to put $c_{j}$ disjoint balls of size $R_{j}$ into $\Omega^{j}$.

Let $\Omega_{d}^{j}=\left\{x \mid\right.$ The distance from $x$ to the complement of $\Omega^{j}$ is $\left.>d=2(3)^{1 / 2} R_{j}\right\}$. Imagine covering $\Omega^{j}$ with a grid of cubes of side $2 R_{j}$. If we throw away all the cubes that touch the complement of $\Omega^{j}$ we will still be able to cover a fraction of the volume of $\Omega^{j}$ that equals or exceeds $\left|\Omega_{d}^{j}\right| /\left|\Omega^{j}\right|$ with cubes. Since we can place one ball in each such cube we have

$$
\# \text { of balls }=\text { \# of cubes } \geqq \frac{\left|\Omega_{d}^{j}\right|}{\left|\Omega^{j}\right|} \cdot \frac{\left|\Omega^{j}\right|}{8 R_{j}^{3}} .
$$

We must show that 


$$
\frac{\left|\Omega_{d}^{j}\right|}{8 R_{j}^{3}} \geqq \frac{|Q|}{M+6} \cdot \frac{1}{\left|B_{R_{J}}\right|},
$$

for once we have this inequality we can throw away what is necessary to put $c_{j}$ in the proper range.

If $\lambda$ is the side length of $Q$ we have

$$
\left|\Omega_{d}^{j}\right| \geqq(\lambda-2 d)^{3}-\sum_{i=j+1}^{M}\left(\frac{\lambda^{3}}{M+5}\left|B_{R_{i}}\right|^{-1}\right)\left|B_{R_{i}+d}\right|
$$

because any point that is more than $d=2(3)^{1 / 2} R_{j}$ from the boundary of $Q$ or more than $R_{i}+d$ from the centre of an existing ball in the covering is guaranteed to be in $\Omega_{d}^{j}$. Thus we will have shown (1) if we can prove

$$
(\lambda-2 d)^{3}-\sum_{i=j+1}^{M}\left(\frac{\lambda^{3}}{M+5} \cdot\left|B_{R_{l}}\right|^{-1}\right)\left|B_{R_{i}+d}\right| \geqq \frac{\lambda^{3}}{M+6} \cdot \frac{8 R_{j}^{3}}{\frac{4}{3} \pi R_{j}^{3}} .
$$

This is equivalent to

$$
\left(1-\frac{4 \sqrt{3} R_{j}}{\lambda}\right)-\frac{1}{M+5} \sum_{i=j+1}^{M}\left(1+2 \sqrt{3} \frac{R_{j}}{R_{i}}\right)^{3} \geqq \frac{6}{\pi}\left(\frac{1}{M+6}\right) .
$$

Elementary calculations show that (2) is true if $|Q| \geqq(12(M+6))^{3}\left|B_{R M}\right|$ and $R_{i+1} \geqq 15 R_{i}$.

The actual thing we will need is the decomposition of some large ball $B_{R}$ into swiss cheese balls. It is easy to construct this decomposition if one first decomposes the large ball into cubes $Q$ and applies the lemma. There is a slight subtlety involved because the boundary of $B_{R}$ will naturally cut across some of the balls and cubes in the covering. We will include in their entirety any balls or cubes that touch the boundary. To get estimates on how many balls of a given size touch $B_{R}$ we first note that for a given $i$ this quantity is bounded below by

$$
\frac{\mid \cup\left(\text { Balls of size } R_{i} \text { in a given } Q_{\lambda}\right) \mid}{\left|Q_{\lambda}\right|} \cdot\left(\text { \# of } Q_{\lambda} \text { that lie entirely in } B_{R}\right. \text { ) }
$$

and bounded above by

$$
\frac{\mid \cup\left(\text { Balls of size } R_{i} \text { in a given } Q_{\lambda}\right) \mid}{\left|Q_{\lambda}\right|} \cdot\left(\text { \# of } Q_{\lambda} \text { that touch } B_{R}\right) \text {. }
$$

Now (\# of $Q$ that lie entirely in $\left.B_{R}\right) \geqq \frac{\left|B_{(R-\sqrt{ } 3 \lambda}\right|}{\left|Q_{\lambda}\right|}$ and (\# of $Q$ that touch $B_{R}$ ) $\frac{\left|B_{(R+\sqrt{ } 3 \lambda)}\right|}{\left|Q_{\lambda}\right|}$. Recall that $\frac{1}{M+6} \leqq \frac{\mid \cup\left(\text { Balls of size } R_{i} \text { in } Q_{\lambda}\right) \mid}{\left|Q_{\lambda}\right|} \leqq \frac{1}{M+5}$, so we have

$$
\begin{aligned}
\frac{1}{M+7} & \leqq \frac{1}{M+6}\left(1-\frac{c \lambda}{R}\right) \leqq \frac{\mid \cup\left(\text { Balls of size } R_{i} \text { that touch } B_{R}\right) \mid}{\left|B_{R}\right|} \\
& \leqq \frac{1}{M+5}\left(1+\frac{c \lambda}{R}\right) \leqq \frac{1}{M+4} .
\end{aligned}
$$


The inequalities all the way to the left and right hold for $R$ sufficiently large compared to $\lambda$, for example $R=c(M+7) \lambda$.

Also, this decomposition misses a tiny fraction of the volume; one can cover that remaining volume with a grid of unit cubes and subsequently dilate the cubes by a factor of two to give a complete covering.

For technical reasons related to the particular potentials we are investigating here, it will be necessary to introduce an ensemble of dilated swiss cheeses consisting of balls of radius $s R_{i}$ where the $R_{i}$ are the radii in the original covering. The values $s$ will vary continuously from some $s_{\min }>1 / 2$ to 1 . Each value of $s$ will induce a particular covering of $\mathbb{R}^{3}$ and of $B_{R}$. It is clear that if the original swiss cheese radii satisfy the condition of the covering lemma the radii in the contracted cheese will still obey the condition because the condition only requires that the sizes of successive radii have a certain minimum ratio: that ratio is preserved under uniform contractions of the radii. All of the above results still hold: we will still have

$$
\frac{1}{M+7} \leqq \frac{\mid \cup\left(\text { Balls of size } s R_{i} \text { that touch } B_{R}\right) \mid}{\left|B_{R}\right|} \leqq \frac{1}{M+4}
$$

for all $s, s_{\min } \leqq s \leqq 1$.

\section{The Exploded System}

Once one has created the swiss cheese it is possible to pull the original system on $B_{R}$ apart into a set of non-interacting subsystems. Suppose a value of $s$ and associated swiss cheese have been fixed. Let us denote each of the elements of the swiss cheese, either a ball or one of the small cubes in the remainder set, by $u_{\gamma(s)}$. To each such element $u_{\gamma(s)}$ we can associate an element $u_{\gamma(s)}^{\uparrow}$ in an "exploded set" consisting of the original elements $u_{\gamma(s)}$ translated by vectors $\xi_{\gamma(s)}$ in such a way that the elements of $\left\{u_{\gamma(s)}^{\uparrow}\right\}$ are disjoint.

On this exploded system one introduces a "phony" Hamiltonian, $H^{\text {phony }}\left(s, N, B_{R}\right)$, acting on some subset of $L_{N^{2}}\left(\cup u_{\gamma(s)}^{\uparrow}\right)$ that has the correct symmetry. $H^{\text {phony }}$ breaks up as a sum of terms on cubes and balls: on cubes the Hamiltonian has only the kinetic energy of the particles in the cube, and on balls the Hamiltonian features kinetic energy and interactions among the particles in the same ball via the $1 / r^{\alpha}$ potential,

$$
H^{\text {phony }}\left(s, N, B_{R}\right)=-\kappa_{1} \Delta_{N_{1}, \cup u_{\gamma(s)}^{\dagger}}-\kappa_{2} \Delta_{N_{2}, \cup u_{\gamma(s)}^{\uparrow}}+\sum_{\text {Balls }} \frac{1}{2} \sum_{i \neq j} \chi_{u_{\gamma(s)}^{\uparrow}}\left(z_{i}\right) \chi_{u_{\gamma(s)}^{\uparrow}}\left(z_{j}\right) \frac{e_{i} e_{j}}{\left|z_{i}-z_{j}\right|^{\alpha}} .
$$

Here the Laplacian terms are understood to be the $N_{i}$ particle Laplacians with Dirichlet boundary conditions on $\left\{\otimes u_{\gamma(s)}^{\uparrow}\right\}$. For convenience we will denote the Laplacian terms $-\Delta_{N \text {, ex }}$.

$H^{\text {phony }}$ is in some sense an approximation to the real Hamiltonian which lives on $B_{R}$. In order to compare $H^{\text {phony }}$ with $H_{N, B_{R}}$ we have to have some method for moving the phony Hamiltonian over the correct Hilbert space. This is accomplished by defining an injection $i(s): L_{N}^{2}\left(B_{R}\right) \rightarrow L_{N}^{2}\left(\cup u_{\gamma(s)}^{\uparrow}\right)$.

To create such an injection we introduce the following partition of unity, $\left\{\phi_{\gamma(s)}\right\}$ : 
1. $\sum_{u_{\gamma(s)}} \phi_{\gamma(s)}^{2}=1$.

2. Each $\phi_{\gamma(s)}$ is supported in a particular $u_{\gamma(s)}$.

3. For the balls, the $\phi_{\gamma(s)}$ are translates of functions $\phi_{i, s}(x)$, where

$\phi_{i, s}(x) \in C_{0}^{\infty}\left(B_{s R_{i}}\right), \quad \phi_{i, s}(x)=1 \quad$ on $B_{\left(s R_{i}-s\right)}$ and $\left|\partial_{x}^{\beta} \phi_{i, s}(x)\right| \leqq C$ for $|\beta| \leqq 3$.

4. For the cubes, the $\phi_{\gamma(s)}$ satisfy $\left|\partial_{x}^{\beta} \phi_{\gamma(s)}(x)\right| \leqq C$ for $|\beta| \leqq 3$.

(Recall that since the cubes in the covering were first chosen to cover the remainder set and then blown up by a factor of two, the cubes overlap all of the balls slightly and act as "glue" to hold everything together and make it possible to satisfy conditions 1 to 3 by making appropriate adjustments in the partition function over each individual cube within the parameters allowed by 4.)

Since any $w \in u_{\gamma(s)}^{\uparrow}$ has a unique expression as $w=z+\xi_{\gamma(s)}$ for some $z \in B_{R}$ we can extend any $\psi \in L_{N}^{2}\left(B_{R}\right)$ to be zero outside $\left(B_{R}\right)^{N}$ and define $i(s) \psi\left(w_{1}, \ldots, w_{|N|}\right)=$ $\psi\left(z_{1}, \ldots, z_{|N|}\right) \prod_{i=1}^{|N|} \phi_{\gamma_{i}(s)}\left(z_{i}\right)$. The injection allows us to pull $H_{\text {phony }}$ back to an operator $H_{N, B_{R}}^{i(s)}=i^{*}(s) \circ H^{\text {phony }}\left(s, N, B_{R}\right) \circ i(s)$ on $L_{N}^{2}\left(B_{R}\right)$.

We note two important facts about this injection. First of all, $i(s)$ is not onto: $i \psi$ must satisfy a compatibility condition due to the fact that more than one point in $\cup u_{\gamma(s)}^{i}$ may correspond to a single $z \in B_{R}$. Secondly, $i(s)$ maps orthonormal sequences to orthonormal sequences. The inner product on the exploded system looks like

$$
\begin{aligned}
& \langle i(s, \tau) \Psi \mid i(s, \tau) \Phi\rangle=\int_{\left(\cup u_{\gamma(s)}^{\dagger}\right)_{i N \mid}} i \Psi\left(w_{1} \cdots w_{|N|}\right) \overline{i \Phi\left(w_{1} \cdots w_{|N|}\right)} d^{3} w_{1} \cdots d^{3} w_{|N|} \\
& =\sum_{\gamma_{1}(s) \cdots \gamma_{|N|}(s)} \int_{u_{\gamma_{1}(s)}^{\dagger} \cdots \cdots \times \int_{\gamma_{|| \mid}(s)}^{\dagger}} i \Psi\left(w_{1} \cdots w_{|N|}\right) \overline{i \Phi\left(w_{1} \cdots w_{|N|}\right)} d^{3} w_{1} \cdots d^{3} w_{|N|} .
\end{aligned}
$$

We use the definition of the injection to write this as an integral on the original Hilbert space:

$$
\sum_{\gamma_{1}(s) \cdots \gamma_{|N|}(s)} \int_{u_{\gamma_{1}(s)} \times} \cdots \int_{\times u_{\gamma_{|N|}(s)}} \Psi\left(z_{1} \cdots z_{|N|}\right) \overline{\Phi\left(z_{1} \cdots z_{|N|}\right)} \prod_{i=1}^{|N|} \phi_{\gamma(s)}^{2}\left(z_{i}\right) d^{3} z_{1} \cdots d^{3} z_{|N|} .
$$

We can take the sum inside the integral and use the fact that an integral of one of the partition functions over an element of the swiss cheese is the same as the integral of that same function over all of $B_{R}$ to get

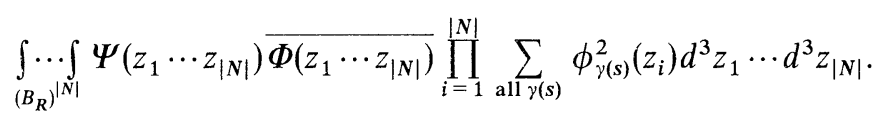

Finally, we use the fact that the sum is exactly one to see that this is exactly the inner product on the original space:

$$
\int_{\left(B_{R}\right)} \cdots \int_{|N|} \Psi\left(z_{1} \cdots z_{|N|}\right) \overline{\Phi\left(z_{1} \cdots z_{|N|}\right) d^{3}} z_{1} \cdots d^{3} z_{|N|}
$$

Thus the injection $i$ preserves inner products and orthogonal sequences get sent to orthogonal sequences. 
These two facts about $i(s)$ combine to give us the important trace inequality

$$
\operatorname{Tr}\left[\exp \left(-\beta H_{N, B_{R}}^{i(s)}\right)\right] \leqq \operatorname{Tr}\left[\exp \left(-\beta H^{\text {phony }}\left(s, N, B_{R}\right)\right)\right] .
$$

We need one more step to facilitate the comparison of $H_{N, B_{R}}$ and $H^{i(s)}$. The latter depends in a certain definite way on the exact nature of the swiss cheese. We can "smooth out" $H^{i(s)}$ by averaging over translations and contractions of the swiss cheese. For each fixed $s$ we can translate the covering of $\mathbb{R}^{3}$ by a set of vectors $\tau$ in some large ball $B_{d}$ whose radius is larger than that of $B_{R}$. This gives rise to a new partition of unity $\left\{\phi_{\gamma(s)}(z-\tau)\right\}$, a new Hilbert space $L_{N}^{2}\left(\cup u_{\gamma(s, \tau)}^{\dagger}\right)$, a new injection $i(s, \tau)$, and a new operator $H^{i(s, \tau)}$. Each new choice for $s$ in turn produces an entirely new set-up. Note, however, that translating the cover does not change the truth of our claims for the properties of $i$ shown above.

The operator we will concentrate on is $H_{N, B_{R}}^{\#}=\operatorname{avg} H_{N, B_{R}}^{i(s, \tau)}$, where $s$ runs over some range $s_{\min } \leqq s \leqq 1$ and $\tau$ runs over all the points in $B_{d}$. It is this operator whose potential we shall compare with the original $H$ via machinery based on the main lemma developed above.

First there will be some preliminary manipulations. By virtue of the convexity of $A \rightarrow \ln \operatorname{Tr}[\exp A]$ and Eq. (1) we have

$$
\ln \sum_{N} e^{\mu \cdot N} \operatorname{Tr}\left[\exp \left(-\beta H_{N, B_{R}}^{\#}\right)\right] \leqq \underset{s, \tau}{\operatorname{avg}}\left\{\ln \sum_{N} e^{\mu \cdot N} \operatorname{Tr}\left[\exp \left(-\beta H^{\text {phony }}\left(s, N, B_{R}\right)\right)\right]\right\} .
$$

Since the elements of the exploded system are disjoint, the partition function for any of the exploded systems decomposes into a product of factors for each of the $u_{\gamma(s, \tau)}^{\dagger}$. We can control the contribution from the cubes by noting that since both the protons and electrons are fermions we have

$$
\operatorname{Tr} \exp \left(\mu \cdot N+\beta \Delta_{N, \mathrm{ex}}\right) \leqq c \exp \left(e^{\mu}\right) \frac{(\text { volume of cube })}{\beta^{3 / 2}}
$$

by the eigenvalue asymptotics of the Laplacian. Thus we can define

$$
\operatorname{Tr} \exp \left(\mu \cdot N+\beta \Delta_{N, \mathrm{ex}}\right) \equiv c(\beta, \mu) \text { (volume of cube). }
$$

The covering lemma shows that the cubes take up a volume in $B_{R}$ that is bounded by $c\left|B_{R}\right| / M$. Thus

$$
\prod_{\text {cubes }} \operatorname{Tr}\left[\exp \left(\beta \Delta_{N, \mathrm{ex}}\right)\right] \leqq \exp \left(\frac{c(\beta, \mu)}{M}\left|B_{R}\right|\right) .
$$

The product over balls can be given a bound independent of $\tau$ because the product only depends on the number of balls of a given radius in the translated covering that intersect $B_{R}$. The number of balls of size $s R_{i}$ in the translated covering that touch $B_{R}$ is bounded above by $\frac{1}{M+4} \cdot \frac{\left|B_{R}\right|}{\left|B_{s R_{i}}\right|} \equiv c_{i}(s)$. Consequently one has an upper bound of the form

$$
\begin{aligned}
& \ln \sum_{N} e^{\mu \cdot N} \operatorname{Tr}\left[\exp \left(-\beta H_{N, B_{R}}^{\#}\right)\right] \\
& \quad \leqq \underset{s}{\operatorname{avg}}\left\{\frac{c(\mu, \beta)}{M}\left|B_{R}\right|+\sum_{i=1}^{M} c_{i}(s) \ln \left(\sum_{N \geqq 0} e^{\mu \cdot N} \operatorname{Tr}\left[\exp \left(-\beta H_{N, B_{s R_{i}}}\right)\right]\right)\right\} .
\end{aligned}
$$


Note that the situation is not entirely satisfactory now because we still have an inequality that contains both $H^{\#}$ and $H$. Our method for replacing the $H^{\#}$ with a quantity that contains $H$ instead is the subject of the next section.

\section{The Main Theorem}

Main Theorem. In the swiss cheese set-up given above if the smallest balls have radius greater than some $R_{\min }$ and the ball $B_{d}$ is large enough we have

$$
H^{\#, B_{R}} \leqq H_{N, B_{R}}+c\left(s_{\min }\right)(1 / M)\left(H_{N, B_{R}}+C\left(N_{1}+N_{2}\right)\right),
$$

where the constant is independent of $N$ and $R$ and

$$
c\left(s_{\min }\right)=C_{1}+C_{2} \cdot\left(s_{\min } /\left(1-s_{\min }\right)\right) .
$$

The idea of the proof is to determine the form of the operator $H^{\#}{ }_{N, B_{R}}$, compare it with that of $H_{N, B_{R}}$, and show that the difference between the two is an operator that is small compared to the Hamiltonian plus the total particle number. The present case closely parallels the original method of Fefferman; for the sake of completeness I shall repeat most of the relevant details. The interested reader is encouraged to look at Fefferman $[1,2]$ for the proof in the Coulomb case.

The first thing to do is to determine the explicit form of both the kinetic and potential energy components of $H_{N, B_{R}}^{\#}$. For the kinetic energy, it is relatively easy to write down the definition of the kinetic energy part of $H^{i(s, t)}$, perform a simple integration by parts and subsequently average over $s$ and $\tau$ to get the kinetic energy part of $H u_{N, B_{R}}^{\#}$. The result looks like the kinetic energy of $H_{N, B_{R}}$ plus an error term whose magnitude is $\underset{s}{\operatorname{avg}}\left(\frac{C}{M} \sum_{j=1}^{|N|} \int|\psi|^{2} d^{3} z_{1} \cdots d^{3} z_{|N|}\right)=\frac{C}{M}|N|$. The factor of $\frac{C}{M}$ comes from the geometric properties of the cutoff functions $\phi_{\gamma_{i}(s)}\left(z_{i}-\tau\right)$ : the fraction of the total volume over which these functions fail to be constant can be bounded above by $\frac{C}{M}$.

Now we turn to the potential energy terms. Averaging the potential energy portion of $H^{i(s, \tau)}$ over $s$ and $\tau$ produces

$$
\int_{\left(B_{R}\right)}\left\{\frac{1}{2} \sum_{i \neq j} e_{i} e_{j} k^{\#}\left(z_{i}, z_{j}\right)\right\}\left|\psi\left(z_{1}, \ldots, z_{|N|}\right)\right|^{2} d^{3} z_{1} \cdots d^{3} z_{|N|},
$$

where

$$
k^{\#}(x, y)=\underset{s}{\operatorname{avg}}\left[|x-y|^{-\alpha} \frac{1}{\left|B_{d}\right|} \int_{B_{d}} \sum_{\left\{u_{\gamma(s)}+\tau\right\} \cap B_{R} \neq \varnothing} \phi_{\gamma(s)}^{2}(x-\tau) \phi_{\gamma(s)}^{2}(y-\tau) d^{3} \tau\right]=\underset{s}{\operatorname{avg}} k^{s}(x, y) .
$$

It is possible to simplify this expression somewhat by noting that the cutoff functions coming from balls of the same size look like translates of the same basic functions. If we isolate a single ball of size $s R_{i}$ from the set $\left\{u_{\gamma(s)}+\tau\right\} \cap B_{R} \neq \varnothing$ for some value of $\tau$ we have for that particular $u_{\gamma(s)}$

$$
\int_{B_{d}} \phi_{\gamma(s)}^{2}(x-\tau) \phi_{\gamma(s)}^{2}(y-\tau) d^{3} \tau=\int_{\mathbb{R}^{3}} \phi_{i, s}^{2}(x-\tau) \phi_{i, s}^{2}(y-\tau) d^{3} \tau .
$$


Since the idea is to replace the protion of the integral over $B_{d}$ that comes from a given $u_{\gamma(s)}$ with the simpler integral over $\mathbb{R}^{3}$, we need to count how many such balls of size $i$ in a given translated covering intersect $B_{R}$.

A relevant fact to recall about the construction of the swiss cheese is that the cheese repeats over length scales the size of the side of the cube $Q$. If one were to consider a point deep inside the ball $B_{R}$ (here "deep inside" means more than $2 \sqrt{2}$ side lengths of $Q$ ) and translate the covering over all $\tau$ in some cube of the roughly the same size as $Q$, that sort of average would be sufficient for our purposes. Unfortunately there are some points near the boundary of $B_{R}$ to take into account as well. If one were to translate the covering over a region the size of $Q$, one would have to worry about what happens to balls in the swiss cheese that originally sat near the boundary of $B_{R}$ : they would spend part of their time over points in $B_{R}$ and the other part of their time over points not contained in $B_{R}$. In order to avoid those sorts of subtleties we are translating over a ball $B_{d}$ whose radius is much larger than $R$. All the balls of size $s R_{i}$ in $B_{d}$ get an opportunity to intersect $B_{R}$ and each ball in the swiss cheese lies over $B_{R}$ for a fraction $\left|B_{R}\right| /\left|B_{d}\right|$ of all of the $\tau$ 's. There is still an error of size $1 / d$ due to balls that lie a distance $d$ from the edge of $B_{R}$, but this error can very easily be absorbed into the error terms $O\left(1 / M^{2}\right)$ below. There are $\frac{\left|B_{d}\right|}{\left|B_{s R_{i}}\right|}\left(\frac{1}{(M+7}+O\left(\frac{1}{M^{2}}\right)\right)$ balls of each size touching $B_{R}$ for a given value of $\tau$, and as we have just noted, translating only changes things in the $O\left(1 / M^{2}\right)$ term. Putting this all together we see that

$$
k^{s}(x, y)=\sum_{i=1}^{M}\left(\frac{1}{M+7}+O\left(\frac{1}{M^{2}}\right)\right)|x-y|^{-\alpha} \frac{\phi_{i, s}^{2} * \phi_{i, s}^{2}(x-y)}{\left|B_{s R_{1}}\right|} .
$$

We would like to apply the main lemma to the potentials $k^{s}(x, y)$ we have generated with our machinery. Since we have only made some general statements about what the $\phi_{i, s}$ should look like we are free to pick a more specific set of $\phi_{i, s}$ to suit our needs. We set $\phi_{i, s}^{2}(x)=\chi\left(|x| \leqq s R_{i}-s / 2\right) * \phi_{s}(x)$ for some $\phi_{s} \in C_{0}^{\infty}(B(0, s / 2))$ and note that these functions satisfy the conditions we placed on the partition of unity functions in Sect. five. Now note that

$$
\frac{\chi\left(|X| \leqq s R_{i}-s / 2\right) * \chi\left(|X| \leqq s R_{i}-s / 2\right)}{\left|B_{s R_{l}-s / 2}\right|}(X)=\chi(|X| \leqq 1) * \chi(|X| \leqq 1)\left(\frac{X}{s R_{i}-s / 2}\right)
$$

has behavior almost like the desired

$$
\frac{\phi_{i . s}^{2} * \phi_{i, s}^{2}}{\left|B_{s R_{i}}\right|}(x-y)=Q_{s}^{i}(X)
$$

The differences can be estimated by noting that $\phi_{i, s}^{2}$ can be trapped between functions $\chi\left(|x| \leqq s R_{i}-s\right)$ and $\chi\left(|x| \leqq s R_{i}+s\right)$. This means that the differences between the $Q_{s}^{i}$ and the convolutions of characteristic functions are fringe effects which will change the estimates of functions and their derivatives by some multiplicative factors that go roughly as the ratio of $\left|B_{s R_{i}-s}\right|$ to $\left|B_{s R_{i}+s}\right|$, or the relative size of the fringe area. Provided that the smallest balls in the covering 
have radii large compared to one this error can be absorbed into the constants below.

From the above equality it is easy to see that the derivatives of the $Q_{s}^{i}(X)$ satisfy

$$
\begin{gathered}
\left|\partial_{x}^{\beta} Q_{s}^{i}(X)\right| \leqq \frac{C}{\left|s R_{i}-s / 2\right|^{|\beta|}} \text { for }|\beta| \leqq 2 \text { and all } X, \\
\text { and for }|\beta|=3 \text { and } X \notin B_{2 s R_{\imath}} \backslash B_{2\left(s R_{1}-s\right)}, \\
\left|\partial_{x}^{\beta} Q_{s}^{i}(X)\right| \leqq C \text { for }|\beta|=3 \text { and } x \in B_{2 s R_{\imath}} \backslash B_{2\left(s R_{1}-s\right)} .
\end{gathered}
$$

In order to get sufficiently nice conditions on the third derivative it is necessary to introduce the average over contractions of the swiss cheese. This will have the effect of smoothing out the $(2-|X|)_{+}^{2}$ singularity in $\chi(|X| \leqq 1) * \chi(|X| \leqq 1)$. It is precisely this singularity which accounts for the fact that $c\left(s_{\min }\right)$ becomes singular when $s_{\min }$ is one. No such smoothing occurred in Fefferman's original proof; instead he used a slightly different main lemma, one which was more forgiving in the third derivative condition for $k(x)$. It turns out that Fefferman's proof can not be generalized in a straightforward way for the case $0<\alpha<1$. Hence the main lemma takes the stricter form that I have used here, and it becomes necessary to introduce an average over contractions of the swiss cheese radii.

Let us examine the functions avg $\left(Q_{s}^{i}(X)\right)$. We have

$$
\underset{s}{\operatorname{avg}} Q_{i}^{s}(X)=\frac{1}{\left(\frac{1}{s_{\min }}-1\right) X} \int_{s_{\min }}^{1} Q_{1}^{i}\left(\frac{X}{\lambda}\right) d \lambda=\frac{s_{\min }}{\left(1-s_{\min }\right) X} \int_{X / s_{\min }}^{X} Q_{1}^{i}(t) d t .
$$

One gets the following estimates for the derivatives of the smoothed functions:

$$
\left|\partial_{x}^{\beta}\left(\underset{s}{\left.\operatorname{avg} Q_{s}^{i}(X)\right)}\right)\right| \leqq\left\{\begin{array}{l}
0 \text { for }|X| \geqq 2 R_{i} \text { and all } \beta \\
\frac{C}{\left(R_{i}-1 / 2\right)^{|\beta|} \cdot \frac{s_{\min }}{1-s_{\min }} \text { for } 1 \leqq|\beta| \leqq 3} \text { all }|X| \leqq 2 R_{i} . \\
C \text { for }|\beta|=0 \text { and all } X
\end{array}\right. \text { and }
$$

Thus each of the terms in $k^{\#}$ satisfies the conditions of the lemma. Unfortunately, there happen to be $M$ terms in all, so we can only get an inequality like

$$
V\left[|X|^{-\alpha}-k^{\#}(X)\right] \geqq-C\left(s_{\min }\right)\left(H_{N, B_{R}}+C\left(N_{1}+N_{2}\right)\right) .
$$

We get the inequality we need by comparing the operators we have with an intermediate operator that is itself easy to understand and control. Introduce

$$
k_{\text {int }}(X)=\underset{s}{\operatorname{avg}}\left(\sum_{i=1}^{M}\left(\frac{1}{M}\right) \phi_{i, s}^{2} * \frac{1}{|X|^{\alpha}} * \phi_{i, s}^{2}(X)\right)=\sum_{i=1}^{M}\left(\frac{1}{M}\right) k_{\text {int }}^{i}(X),
$$

and examine $V\left[|X|^{-\alpha}-k^{\sharp}(X)-k_{\text {int }}(X)\right]$. To facilitate this comparison we write

$$
|X|^{-\alpha}-k^{\#}(X)=\frac{1}{M} \sum_{i=1}^{M} k_{\dagger}^{i}(X)+\frac{1}{M}|X|^{-\alpha}\left(\sum_{i=1}^{M}\left(1-Q_{0}^{i}\right)\right)+\sum_{i=1}^{M} O\left(\frac{1}{M^{2}}\right) \underset{s}{\operatorname{avg} Q_{s}^{i}(X),}
$$


where

$$
\begin{aligned}
Q_{0}^{i} & =\left.\underset{s}{\operatorname{avg}} \frac{\phi_{i, s}^{2} * \phi_{i, s}^{2}(X)}{\left|B_{s R_{i}}\right|}\right|_{X=0} \\
& \geqq \underset{s}{\operatorname{avg}} \int_{\mathbb{R}^{3}} \frac{\chi\left(|X| \leqq s R_{i}-s\right) \cdot \chi\left(|X| \leqq s R_{i}-s\right)}{\left|B_{s R_{i}}\right|} d^{3} X \\
& =\underset{s}{\operatorname{avg}} \frac{\left|B_{\left(s R_{i}-s\right)}\right|}{\left|B_{s R_{i}}\right|} \geqq 1-\frac{C}{R_{i}},
\end{aligned}
$$

so $\sum_{i=1}^{M}\left(1-Q_{0}^{i}\right) \leqq \sum_{i=1}^{M}\left(C / R_{i}\right) \leqq C$ since the smallest balls in the covering have radii large comparable to unity.

Some comment concerning exactly what is going on here is in order. The image to keep in mind is that $k^{\#}$ looks roughly like $|x|^{-\alpha}$ multiplied by a "staircase function" which is obtained by adding up a series of $M$ functions which look roughly like $(1 / M) \chi\left(|x|<s R_{i}\right) * \chi\left(|x|<s R_{i}\right)$ for $1 \leqq i \leqq M$. This picture is not perfect. In particular, the staircase function is not exactly one at the origin, and the various cut-offs that are averaged together to make the staircase function do not all have the same weights: they actually have weights that are based on what fraction of the total volume of $B_{R}$ is occupied by balls of size $i$. The correction term containing the factors $Q_{0}^{i}$ was thrown in so that $k_{+}$would vanish at the origin. The second correction term exists to absorb the fluctuations in the volume fractions and thus give all of the terms in $k_{\dagger}$ the same weight.

It turns out that $k_{\dagger}^{i}-k_{\text {int }}^{i}$ has the right behavior, although there is a slight difference between the cases $\alpha<1$ and $\alpha>1$. Consider first the case $\alpha>1$ :

1. As $X \rightarrow 0, R_{i}|X|^{\alpha-1} k_{\dagger}^{i}(X) \rightarrow 0$ and $k_{\text {int }}^{i}(X) \leqq C / R_{i}^{\alpha}$, where $C$ can be chosen independent of $s_{\min }$.

2. Out near $|X|=s_{\min } R_{i}, R_{i}^{\alpha} k_{+}^{i}(X)=c \cdot s_{\min }^{-\alpha} \leqq C$ and $R_{i}^{\alpha} k_{\text {int }}^{i}(X)=c \cdot s_{\min }^{-\alpha} \leqq C$, where both $C$ can be taken independent of $S_{\min }$.

3. In between these two values of $|X|$ the functions $R_{i}|X|^{\alpha-1} k_{\dagger}^{i}(X)$ and $R_{i}|X|^{\alpha-1} k_{\text {int }}^{i}(X)$ can both be bounded above by some constant $C$ which is independent of both $i$ and $s_{\min }$.

This produces the nice derivative estimate

4. $\left|\partial_{x}^{\beta}\left(k_{\dagger}^{i}(X)-k_{\mathrm{int}}^{i}(X)\right)\right| \leqq \frac{C}{R_{i}} \cdot \frac{1}{|X|^{|\beta|+\alpha-1}}\left(\frac{s_{\min }}{1-s_{\min }}\right)$ for $|X| \leqq s_{\min } R_{i}$.

In the case $\alpha<1$ we have

5. As $X \rightarrow 0, k_{+}^{i}(X)=0$ and $k_{\text {int }}^{i}(X) \leqq C / R_{i}^{\alpha}$, where $C$ can be chosen independent of $s_{\min }$.

6. Out near $|X|=s_{\min } R_{i}, R_{i}^{\alpha} k_{+}^{i}(X)=c \cdot a_{\min }^{-\alpha} \leqq C$ and $R_{i}^{\alpha} k_{\text {int }}^{i}(X)=c \cdot s_{\min }^{-\alpha} \leqq C$, where both $C$ can be taken independent of $S_{\min }$.

7. In between these two values of $|X|$ the functions $R_{i}^{\alpha} k_{\dagger}^{i}(X)$ and $R_{i}^{\alpha} k_{\text {int }}^{i}(X)$ can both be bounded above by a constant $C$ which is independent of $i$ and $s_{\min }$. 
This case has derivative estimate

$$
\text { 8. }\left|\partial_{x}^{\beta}\left(k_{+}^{i}(X)-k_{\text {int }}^{i}(x)\right)\right| \leqq \frac{C}{R_{i}^{\alpha}} \cdot \frac{1}{|X|^{|\beta|}}\left(\frac{s_{\min }}{1-s_{\min }}\right) \text { for }|X| \leqq s_{\min } R_{i} \text {. }
$$

Out beyond the neighborhood of the origin we can get away with the following somewhat cruder estimates:

9. $\left|\partial_{x}^{\beta}\left(k_{+}^{i}(X)-k_{\mathrm{int}}^{i}(X)\right)\right| \leqq \frac{C}{|X|^{\alpha+|\beta|}}\left(\frac{s_{\min }}{1-S_{\min }}\right)$ for $s_{\min } R_{i} \leqq|X| \leqq 2 R_{i}$ and $|\beta| \leqq 3$.

10. For $|X| \geqq 2 R_{i}, Q_{s}^{i}(X)=0$, so $k_{\dagger}^{i}(X)=\frac{Q_{0}^{i}}{|X|^{\alpha}}$. Also $k_{\text {int }}^{\mathrm{i}}(X)=\left(1+O\left(\frac{R_{i}^{2}}{|X|^{2}}\right)\right)|X|^{-\alpha}$,

$$
\text { so } \mid \partial_{x}^{\beta}\left(k_{\dagger}^{i}(X)-k_{\text {int }}^{i}(X)\right) \leqq O\left(\frac{R_{i}^{2}}{|X|^{2}}\right) \cdot \frac{1}{|X|^{\alpha+|\beta|}}+O\left(\frac{1}{M}\right) \frac{1}{|X|^{\alpha+|\beta|}} \text { for }|\beta| \leqq 3 \text {. }
$$

These hold for all $\alpha$ in the range of interest. The important thing to note here is the fact that the intervals $\left(s_{\mathrm{sim}} R_{i}, 2 R_{i}\right)$ are all disjoint. Given that the swiss cheese radii satisfy $R_{i+1} \geqq \max \left\{15,2^{1 / \alpha}\right\} R_{i}$ we can take a sum over $i$ in the inequalities 4,9 , and 10 for $\alpha>1$ or a sum over $i$ in 8,9 , and 10 for $\alpha<1$ to get

$$
\left|\partial_{x}^{\beta}\left(k_{\dagger}(X)-k_{\text {int }}(X)\right)\right| \leqq \frac{C}{|X|^{\alpha+|\beta|}}\left(\frac{s_{\min }}{1-s_{\min }}\right)\left(\frac{1}{M}\right)
$$

in both cases, independent of whether $\alpha$ is less than or greater than one.

An application of the lemma gives $V\left[k_{\dagger}-k_{\mathrm{int}}\right] \geqq-\frac{C\left(s_{\min }\right)}{M}\left(H_{N, B_{R}}+C\left(N_{1}+N_{2}\right)\right)$, so that

$$
V\left[|X|^{-\alpha}\right]-V\left[k^{\#}\right] \geqq \frac{C}{M} V\left[|X|^{-\alpha}\right]+V\left[k_{\dagger}(X)\right]+\sum_{i=1}^{M} O\left(\frac{1}{M^{2}}\right) V\left[|X|^{-\alpha}-k_{i}^{\#}(X)\right] .
$$

Using estimate (1) to control the final summation we see that this is

$$
\begin{aligned}
\geqq & \frac{C}{M} V\left[|X|^{-\alpha}\right]-\frac{C\left(s_{\text {min }}\right)}{M}\left(H_{N, B_{R}}+C\left(N_{1}+N_{2}\right)\right) \\
& +V\left[k_{\dagger}(X)-k_{\text {int }}(X)\right]+V\left[k_{\text {int }}(X)\right] \\
\geqq & \frac{C}{M} V\left[|X|^{-\alpha}\right]-\frac{C\left(s_{\text {min }}\right)}{M}\left(H_{N, B_{R}}+C\left(N_{1}+N_{2}\right)\right) \\
& -\frac{C\left(s_{\text {min }}\right)}{M}\left(H_{N, B_{R}}+C\left(N_{1}+N_{2}\right)\right)+V\left[k_{\text {int }}(X)\right] .
\end{aligned}
$$

The only term that remains to be controlled is $V\left[k_{\text {int }}(X)\right]$. This potential is very easy to understand because $|X|^{-\alpha}$ has positive Fourier transform; up to self energy terms that go as $\frac{1}{M} \sum_{i=1}^{M}\left(N_{1}+N_{2}\right) \frac{C}{R_{i}^{\alpha}} \leqq \frac{C}{M}\left(N_{1}+N_{2}\right)$ the operator $V\left[k_{\text {int }}\right]$ is posi- 
tive. So finally

$V\left[|X|^{-\alpha}\right]-V\left[k^{\#}\right] \geqq \frac{C}{M} V\left[|X|^{-\alpha}\right]-\frac{C\left(s_{\min }\right)}{M}\left(H_{N, B_{R}}+C\left(N_{1}+N_{2}\right)\right)-\frac{C}{M}\left(N_{1}+N_{2}\right)$

and

$$
V\left[k^{\#}\right] \leqq V\left[|X|^{-\alpha}\right]+\frac{C\left(s_{\min }\right)}{M}\left(H_{N, B_{R}}+C\left(N_{1}+N_{2}\right)\right) .
$$

Putting this together with the kinetic energy results completes the proof of the main theorem.

\section{The Thermodynamic Limit}

With $M$ chosen so large that $c\left(s_{\min }\right) / M \leqq \varepsilon$ one can substitute the conclusion of the theorem into Eq. (5.2) and obtain

$$
\begin{aligned}
\ln & \sum_{N} e^{(\mu-\bar{\varepsilon}) \cdot N} \operatorname{Tr}\left[\exp \left(-\beta(1+\varepsilon) H_{N, B_{R}}\right)\right] \\
& \leqq \underset{s}{\operatorname{avg}}\left\{c^{\prime}(\beta, \mu) \cdot \varepsilon\left|B_{R}\right|+\sum_{i=1}^{M} c_{i}(s)\left(\ln \sum_{|N| \geqq 0} e^{\mu \cdot N} \operatorname{Tr}\left[\exp \left(-\beta H_{N, B_{s R_{i}}}\right)\right]\right)\right\} .
\end{aligned}
$$

Here $\bar{\varepsilon}=(\varepsilon, \varepsilon)$. The next ingredient we will use is

$$
\begin{aligned}
& \ln \sum_{N} e^{\mu \cdot N} \operatorname{Tr}\left[\exp \left(-\beta H_{N, B_{R}}\right)\right] \\
& \quad \leqq\left\{c^{\prime \prime}(\beta, \mu) \cdot \varepsilon\left|B_{R}\right|+\ln \sum_{N} e^{(\mu-\bar{\varepsilon}) N} \operatorname{Tr}\left[\exp \left(-\beta(1+\varepsilon) H_{N, B_{R}}\right)\right]\right\} .
\end{aligned}
$$

Inequality (2) is equivalent to the Lipschitz continuity of the pressure $\Pi_{R}(\beta, \mu)$. This can be shown by the following:

1. $\Pi_{R}(\beta, \mu)$ is convex in both variables.

2. $\Pi_{R}(\beta, \mu)$ is bounded below: it is easy to cook up an $N$ particle wave function $\left(|N|<c\left|B_{R}\right|\right)$ for which $\left\langle H_{N, B_{R}} \psi \mid \psi\right\rangle \leqq c|N|$. Thus $\Pi_{R}(\beta, \mu)=\frac{1}{\left|B_{R}\right|} \ln \sum_{N} e^{\mu \cdot N} \times$

$$
\operatorname{Tr}\left[\exp \left(-\beta H_{N, B_{R}}\right)\right] \geqq \frac{1}{\left|B_{R}\right|} \ln \left[e^{\mu \cdot N-\beta c|N|}\right] \geqq-c^{\prime}(\beta, \mu) .
$$

3. $\Pi_{R}(\beta, \mu)$ can be bounded above by using the stability of matter inequality in the form $-\frac{1}{2} \Delta_{N}+V\left[|x|^{-\alpha}\right] \geqq-C|N|$ which is a trivial consequence of the short range inequality from Sect. 4. This implies that $\Pi_{R}(\beta, \mu) \leqq$ $\frac{1}{\left|B_{R}\right|} \ln \sum_{N} e^{\mu \cdot N} e^{c \beta|N|} \operatorname{Tr} \exp \left(\frac{\beta}{2} \Delta_{N}\right) \leqq c^{\prime \prime}(\beta, \mu)$.

Putting (1) and (2) together gives the desired inequality 


$$
\begin{aligned}
& \ln \sum_{N} e^{\mu \cdot N} \operatorname{Tr}\left[\exp \left(-\beta H_{N, B_{R}}\right)\right] \\
& \quad \leqq\left\{C(\beta, \mu) \cdot \varepsilon\left|B_{R}\right|+\underset{s}{\operatorname{avg}}\left(\sum_{i=1}^{M} c_{i}(s) \ln \sum_{N \geqq 0} e^{\mu \cdot N} \operatorname{Tr}\left[\exp \left(-\beta H_{N, B_{S R_{i}}}\right)\right]\right)\right\} .
\end{aligned}
$$

Finally, one can make this a statement about pressures by noting that $\frac{c_{i}(s)}{\left|B_{R}\right|} \leqq \frac{1}{M+4} \cdot \frac{1}{\left|B_{s R_{l}}\right|}$ and thus $\Pi_{R}(\beta, \mu) \leqq C(\beta, \mu) \cdot \varepsilon+\sum_{i=1}^{M} \frac{1}{M+4} \underset{s}{\operatorname{avg}} \Pi_{s R_{i}}(\beta, \mu)$.

For the purpose of proving the thermodynamic limit, it is more revealing to average the left-hand side of the last inequality over contractions and rewrite the

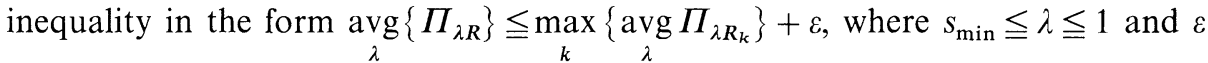
depends on $M$ and $s_{\min }$ via $c\left(s_{\min }\right) / M$.

Claim. This inequality, combined with the fact that $\Pi_{R}$ is bounded below (statement 2 above), is enough to prove that $\lim _{R \rightarrow \infty} \underset{\lambda}{\operatorname{avg}}\left\{\Pi_{\lambda R}\right\}=\Pi_{\infty}$ exists. To see this, let $\varepsilon>0$ be given. First pick $M$ sufficiently large that $c\left(s_{\min }\right) / M<\varepsilon$, and then choose any legitimate sequence of radii $\left\{R_{k}\right\}$. These radii induce an $R^{1}$ such that avg $\left\{\Pi_{\lambda R}\right\} \leqq$ $\max _{k}\left\{\underset{\lambda}{\operatorname{avg}} \Pi_{\lambda R_{k}}\right\}+\varepsilon$ for all $R>R^{1}$. Now there are two alternatives: (1) there exists a new sequence $\left\{R_{k^{\prime}}\right\}$ of swiss cheese radii chosen from $R>R^{1}$ for

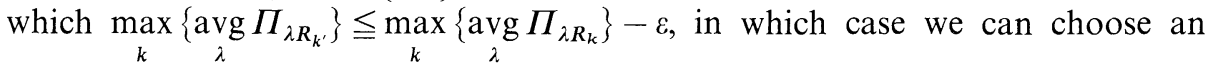
$R^{2}>R^{1}{ }^{k}$ and go through the loop again or (2) $\max _{k}\left\{\underset{\lambda}{\operatorname{avg}} \Pi_{\lambda R_{k}}\right\}-\varepsilon \leqq \underset{\lambda}{\operatorname{avg}}\left\{\Pi_{\lambda R}\right\} \leqq$ $\max \left\{\operatorname{avg} \Pi_{\lambda R_{k}}\right\}+\varepsilon$ for all $R$ beyond some $R_{\text {critical }}$, in which case we are done. Finally, one can use (3) with very large $R>R_{\text {critical }}$ and correspondingly large swiss cheese balls chosen from $R>R_{\text {critical }}$ to show that $\Pi_{R} \leqq \Pi_{\infty}+2 \varepsilon$ for $R$ sufficiently large.

To show that $\Pi_{R}$ itself has a limit we need to prove that for $R$ large we have $\Pi_{R}>\Pi_{\infty}-2 \varepsilon$. Suppose to the contrary that we can find a sequence $\left\{R_{j}\right\}$ of radii going to infinity such that $\Pi_{R_{J}}<\Pi_{\infty}-2 \varepsilon$ for all $j$. We have at our disposal an infinite sequence of these radii, so we are free to select a subsequence of radii such that the values $R_{j}$ obey the requirements for swiss cheese radii. We are also free to pick any number of these radii for use in a special swiss cheese. Let us choose $M$ radii where $M$ is so large that even if we choose $s_{\min }=1-\delta$ for some small $\delta$ to be specified we can still have $c\left(s_{\min }\right) / M<\varepsilon / 100$ in the main theorem. Now consider one of the $R_{j}$. For $(1-\delta) R_{j} \leqq R \leqq R_{j}$ it is easy to show that $\Pi_{R}<\Pi_{\infty}-\varepsilon$ :

1. By minimax $\operatorname{Tr}\left[\exp \left(-\beta H_{N, B_{R}}\right)\right] \leqq \operatorname{Tr}\left[\exp \left(-\beta H_{N, B_{R}}\right)\right]$.

2. $\Pi_{R}=\left(\frac{\left|B_{R_{j}}\right|}{\left|B_{R}\right|}\right) \frac{1}{\left|B_{R_{j}}\right|} \ln \operatorname{Tr}\left[\exp \left(\mu \cdot N-\beta H_{N, B_{R}}\right)\right]$

$$
\leqq\left(\frac{\left|B_{R_{j}}\right|}{\left|B_{R}\right|}\right) \Pi_{R_{j}} \leqq(1-\delta)^{-3} \Pi_{R_{j}} \leqq(1-\delta)^{-3}\left(\Pi_{\infty}-2 \varepsilon\right) \text {. }
$$


Thus for this special swiss cheese we have

$$
\begin{aligned}
\underset{\lambda}{\operatorname{avg}} \Pi_{\lambda R} & \leqq \max _{k}\left\{\underset{\lambda}{\operatorname{avg}} \Pi_{\lambda R_{k}}\right\}+\varepsilon / 100 \\
& \leqq(1-\delta)^{-3}\left(\Pi_{\infty}-2 \varepsilon\right)+\varepsilon / 100 \leqq \Pi_{\infty}-\varepsilon
\end{aligned}
$$

for $\delta$ sufficiently small, which contradicts our original assertion that $\lim _{R \rightarrow \infty} \underset{\lambda}{\operatorname{avg}}\left\{\Pi_{\lambda R}\right\}=\Pi_{\infty}$. Hence there can be no sequence of bad radii, and the pressure goes to a finite limit as $R \rightarrow \infty$.

\section{References}

1. Fefferman, C.: The thermodynamic limit for a crystal. Comm. Math. Phys. 98, 289-311 (1985)

2. Fefferman, C.: The atomic and molecular nature of matter. Rev. Math. Iberoamericana 1, 1-14 (1985)

3. Fefferman, C., de la Llave, $R$ : Relativistic stability of matter, I. Rev. Math. Iberoamericana 2 , 119-215 (1986)

4. Hughes, W.: Thermodynamics for coulomb systems: a problem at vanishing density (to appear)

5. Lebowitz, J., Lieb, E: The constitution of matter: existence of thermodynamics for systems composed of electrons and nuclei. Adv. Math. 9, 316-398 (1972)

6. Reed, M., Simon, B.: Methods of modern mathematical physics: function analysis, Vol. 1. New York: Academic Press 1980

7. Ruelle, D.: Statistical mechanics: rigorous results. New York: W. A. Benjamin 1969

8. Stein, E. M.: Singular integrals and differentiable properties of functions. Princeton, NJ: Princeton University Press 1970

Communicated by M. Aizenman

Received April 19, 1988; in revised form November 11, 1988 